\title{
The Research on STL Parallel Topological Reconstruction Method Based on OpenMP
}

\author{
Huijiang Zheng ${ }^{1,3}$, Jing Zhang ${ }^{2}$, Ying Tian $^{1} \&$ Baihui Ding ${ }^{1}$ \\ ${ }^{1}$ Tianjin Key Lab. for Advanced Manufacturing Tech. \& Equipment, Tianjin University, Tianjin \\ 300072, China \\ ${ }^{2}$ School of Control \& Mechanical Engineering, Tianjin Chengjian University, Tianjin 300384, China \\ ${ }^{3}$ School of Mechanical \& Aerospace Engineering, Queen's University Belfast, Belfast BT9 5AH, UK
}

Keywords: Topological Reconstruction, OpenMP, STL, Parallel-hashing Structure.

\begin{abstract}
The STL(Stereo Lithography) format is one of the data interface format is commonly used in modern manufacturing field. Using parallel-hashing structure as the auxiliary data structure, by adjusting the mapping and reference relationship between each date lists, this paper put forward the parallel topological reconstruction method based on OpenMP. By effectively improving the efficiency of processing hash function conflict and reducing the concurrent access conflict, this method completed parallel merging and topological relation reconstruction to redundant data in STL format file. The calculation examples demonstrated the effectiveness of the proposed method.
\end{abstract}

\section{Introduction}

Because the STL file format has the simple format and is easy to read[1], it is often used to express and transfer all kinds of complex surface parts surface information. It has become one of the commonly used data format in the manufacturing technology field[2,3]. But the STL format file does not contain the constitute information of part surfaces and the basic topological relations between various geometry elements in the triangular mesh[3,4], and data redundancy phenomenon is serious. The high precision triangulate to the surfaces of part with complex structure will produce a large number of triangular mesh models. Therefore need rapidly merge a large number of redundant data in the STL file and accurately reconstruct the topology information between various geometric elements.

In order to improve the efficiency of filtering redundancy data in triangular mesh model and topological information reconstruction, many domestic and foreign scholars have done a lot of research[2-5]. Because of various algorithms are studied under single-core environment, processing for large amount of data could obviously decreased the efficiency of these algorithms. The emergence of Multi-core processors improved the efficiency of the processor for processing data[6], and realized the parallel execution of multiple threads on physics. OpenMP is an application programming interface designed for sharing memory environment and writing parallel program[7]. OpenMP can provide an abstract description of the parallel algorithm. The compiler can automatically parallelize the program, and put in the mutual exclusion synchronization and communication where necessary[8].

Under multi-core computing environment, this paper uses OpenMP2.0 as the main development tool and parallel-hashing table structure as the auxiliary data structure. By adjusting the access strategy to reduce the probability the concurrent access conflicts, propose a method of fast information filtering and topological information reconstruction to STL format file under multi-core environment. This method can effectively improve the computation efficiency of the topology reconstruction.

\section{Build the date structure of parallel-hashing}

Build the hash date structure. If establish a $f$ mapping rule between the geometric elements scalar values and the non repetitive list index values, such that

$$
f: \boldsymbol{R}^{3} \rightarrow N
$$


There is and satisfy $\forall \boldsymbol{A}_{1}\left(a_{1}, b_{1}, c_{1}\right), \boldsymbol{A}_{2}\left(a_{2}, b_{2}, c_{2}\right) \in \boldsymbol{R}^{3}$, if $\boldsymbol{A}_{1} \neq \boldsymbol{A}_{2}$, then $f\left(\boldsymbol{A}_{1}\right) \neq f\left(\boldsymbol{A}_{2}\right)$ and the $f$ rule known as the Hash function[9]. To query by Hash function, need to calculate objects images only once, can judge whether the object is repeated. Generally $\mathrm{f}$ hash mapping exists hash conflict, that is to say, there are many different geometric element scalars corresponding to the same index value. Obviously, the key of the hash structure query efficiency is to build a hash function $f[1,9]$.

By the date structure of parallel-hashing, this paper uses the $n$ triangle number contained in triangular mesh as basic data, with (2) to calculate the $T$ length of Hash table:

$$
T=2^{\left\lfloor\log _{2} n\right\rfloor}-1
$$

In the process of merging geometric element, compute the scalar value as the $K$ positive integer critical value. Floating-point comparison is converted to integer comparison. The formula of the $K$ critical value and the $f$ Hash function is as follows:

$$
\begin{gathered}
K=\operatorname{int}\left(\frac{\xi \operatorname{int}(|x| S)}{S}+\frac{\rho \operatorname{int}(|y| S)}{S}+\frac{\lambda \operatorname{int}(|z| S)}{S}\right) T \\
f(K)=K \bmod T
\end{gathered}
$$

Among them, $x, y, z$ are geometric element scalar value components. $\xi 、 \rho 、 \lambda$ are Hash function coefficients. $S$ is a sensitivity coefficient of Hash function. The mod symbol expresses the remainder computation. Among them, as far as possible choose the prime or different irrational number as $\xi 、 \rho 、 \lambda$, in order to ensure $K$ value the greatest dispersion[1]. Obviously under the multi-core computing environment, it can further improve the retrieval efficiency that set up multiple $f$ computation threads to calculate and to query the $K$ critical value and the $f$ hash function. Because multi thread search exist access competition conflict[7,9], solving the conflict is the key to making parallel hash structure to effectively realize.

Solve the concurrent access conflicts. Using serial hash table structure to process topological relation reconstruction, that is essentially to pick out the nont-repeating geometric elements, and store to sequential array with hash table, from the geometric element arrays stored a large number of disorder and repeated data. The $f(K)$ value of hash function obtained by above method is index value mapped to the hash table from elements in the disorder geometry element array. At the same time, the sequence index value of geometry element in the non repetitive list is recorded in the corresponding element of the hash table. Using vertex table as an example, the mapping relationship is shown in Fig. 1.

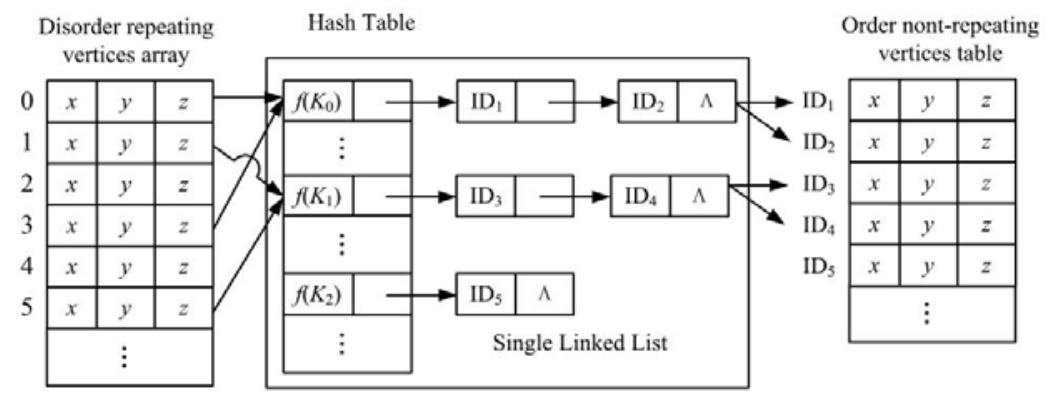

Fig.1: Repeat disorder point table, the hash table and the mapping relationship between the order nont-repeating tables

This paper uses the hash child table method as shown in Fig. 2 to improve the efficiency of concurrent access to the hash table. According to the number of computing core in computer CPU, the original single hash table is divided into the same number of hash table section. The sum of element number from all hash table section equal element number in the original single hash table, and the number of elements in each hash table section is same, to maintain the same storage space with the original hash table. This method defines a data lock for every hash table section to ensure that at any time up to only one thread accesses the child table. 


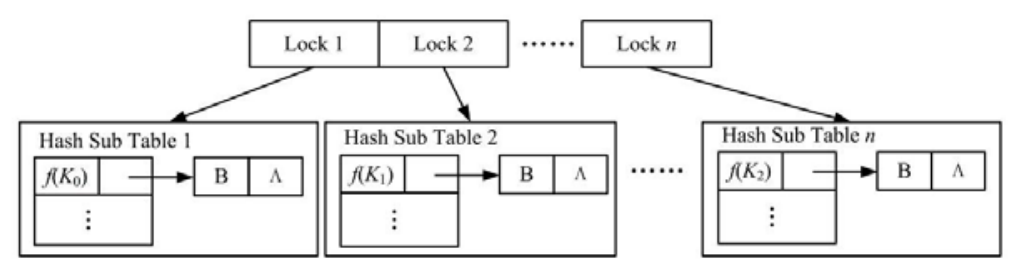

Fig.2: Subsection lock hash table structure schematic diagram

But a number of hash child tables broke the sequence structure in the original hash table structure. Each child table has its own index value sequence. Geometric element array still maintained the original way of the order list, which would require changing mapping relationship in the geometric element table from Fig. 1 to Fig. 3.
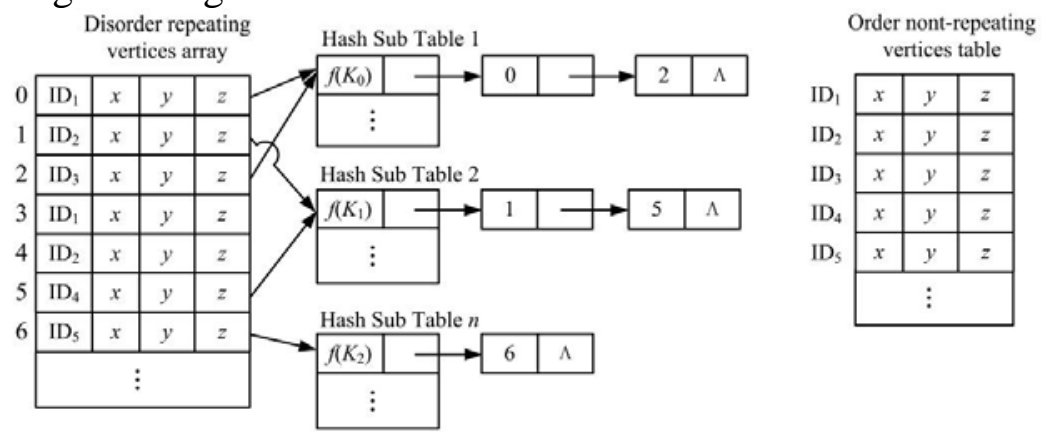

Fig.3:Repeat disorder point table, the parallel-hashing table and the mapping relationship between the order nont-repeating tables

In order to improve the efficiency of concurrent access, the hash table in Fig. 3 no longer record geometric element index values corresponding to order nont-repeating tables of geometric elements. It changes to record the index value of the first repeat value in the array of repeat records, and write the index value of the first repeat value in the nont-repeating table to the first repeat value. In the parallel-hashing method running process, because no repeated geometric element array is dynamic array, it is accessed by multi thread to add new geometric elements. Therefore the array will be repeatedly lock and unlock. If the process of hash value conflict also need access this array, it will increase the chance of concurrent access conflicts, and cause the access efficiency drop. But the repeated element array is the fixed length array. In the array, each element has been accessed by only one thread, and elements which have been accessed will not be written new data. So access to this array will not cause concurrent access conflicts.

Because the OpenMP compiler uses the "Fork/Join" mode to realize the parallel program[7,8]. The parallel part can be realized through the derived on the main thread, so we can use the same code to realize the uniform implementation process of the parallel parts. Using the vertex list merge as an example, in the process of multi thread reconstruction, Fig. 4 shows one thread execution process.

\section{The calculation example and method analysis}

This paper designed five kinds of different part structures by using Pro/e software, and saved as the triangular mesh data to different amount of data, as shown in Fig.5. By the serial hash structure method and the parallel-hashing structure method, the topological information reconstruction was respectively calculated to triangular mesh data are available. Computing environment is as follows: Visual C++2008; OpenMP 2.0; OS is Windows 7 64bit SP1; memory capacity is 8GB; and CPU is i7 $2.7 \mathrm{GHz}$. Table 1 is the results of using two kinds of method to topological reconstruct for different models. 


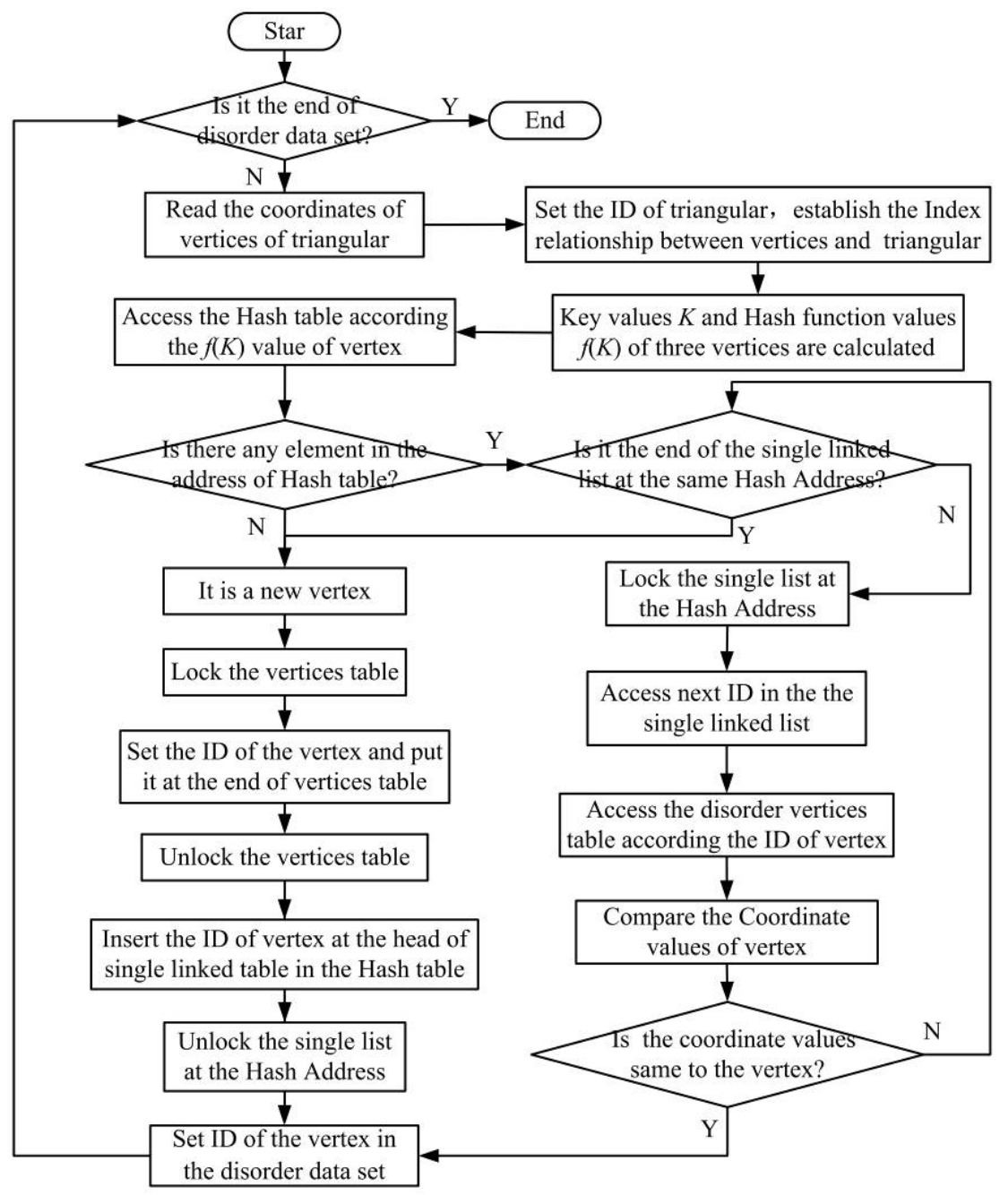

Fig.4: The execution process for one of the multi thread based on OpenMP

The results of example show that the time of topological reconstruction by parallel hashing method is much smaller than by the serial hash method, in dual-core mode. By comparing the ratio of the number of handled triangles in the STL format file and the reconstruction time, it is not difficult to see that the parallel-hashing structure method based on OpenMP basically maintains the character that the hash structure time complexity is $O(n)$. In addition to the ratio of calculation time for the first group less than 1.8, the other four groups averaged 1.901. It showed that with the increase of data quantity, the ratio of occupied time of the data locking operation is relatively stable, most operations are parallel computing, the stability of efficiency advantage of the parallel-hashing structure method based on OpenMP increase.

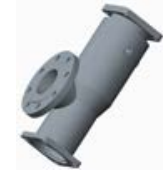

(a) Pump Body

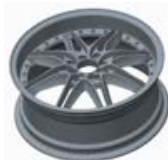

(b)Hub

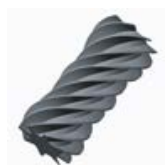

(c) Worm

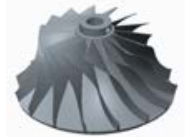

(d) Blade

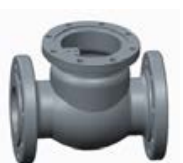

(e) Valve Body

Fig. 5: STL format files used as calculation examples 
Table 1: Results of topological reconstruction for STL format files

\begin{tabular}{|c|c|c|c|c|c|c|}
\hline $\begin{array}{l}\text { STL format } \\
\text { file }\end{array}$ & $\begin{array}{l}\text { the size of } \\
\text { files(Byte) }\end{array}$ & $\begin{array}{l}\text { the } \\
\text { amount } \\
\text { of } \\
\text { triangles }\end{array}$ & $\begin{array}{l}\text { the } \\
\text { amount } \\
\text { of } \\
\text { vertexs }\end{array}$ & $\begin{array}{l}\text { the serial } \\
\text { method } \\
\text { (ms) }\end{array}$ & $\begin{array}{l}\text { the } \\
\text { parallel-hashing } \\
\text { method } \\
\text { (ms) }\end{array}$ & $\begin{array}{l}\text { the ratio of } \\
\text { calculation } \\
\text { time }\end{array}$ \\
\hline Pump Body & $14,550,731$ & 56238 & 28077 & 203.24 & 115.219 & 1.763945 \\
\hline Hub & $29,019,539$ & 111632 & 55766 & 449.025 & 237.952 & 1.88704 \\
\hline Worm & $57,403,826$ & 223396 & 111700 & 950.29 & 496.463 & 1.91412 \\
\hline Blade & $119,009,090$ & 457124 & 228512 & 2040.719 & 1064.553 & 1.916973 \\
\hline Valve Body & $231,546,864$ & 889392 & 444642 & 3671.097 & 1944.943 & 1.887509 \\
\hline
\end{tabular}

\section{Conclusion}

Under the OpenMP2.0 development environment, this paper set up multi core environment to effectively support for parallel hash data structure calculation method, and proposed STL parallel topology reconstruction method based on OpenMP. By improving the Hash function dispersion, this method adjust the establishment method of all kinds of topological structure index value, effectively reduced the probability of concurrent access conflict, and realized redundant data in the STL format files were quickly merge and topological information reconstruction. By using different methods for different amounts of data of STL format file, the result of calculation example contrast verified the efficiency and effectiveness of the present method.

\section{Acknowledgements}

The research work was supported by National Natural Science Foundation of China under Grant No.51105271, State Scholarship Fund supported by the China Scholarship Council under Grant No.20140625508 and Science and Technology Development Foundation for Colleges and universities of Tianjin under Grant No.20130409. Huijiang Zheng is the correspondent of this paper.

\section{References}

[1] Jan Hradek, Martin Kuchar, Vaclav Skala. Hash functions and triangular mesh reconstruction[J]. Computers \& Geosciences, 2003, 29(6):741-751.

[2] Zhu Hu, Yang Xiaoguang. A Study on the Interference Detection and Correction in 5-Axis CNC Incremental Forming [J]. Journal of Mechanical Engineering, 2014, 50(7):168-174.

[3] Zheng Huijiang. Research on the Key Technology of the Topological Feature Reconstruction and Sampling Point Distribution in OMV[D]. Tianjin: Tianjin University,2010.

[4] Szilvási-Nagy M , Mátyási G. Analysis of STL files[J]. Mathematical and Computer Modeling, 2003, 38 (7):945 960.

[5] Yang Shengyuan, Shu Shi. Fast Reconstruction of STL Surface Meshes Based on Data Correlation[J]. Journal of Computer-Aided Design \& Computer Graphics, 2009, 21(1):67 71.

[6] Chen Yongheng, Zuo Xianglin. Parallel Two-Way Enumeration Join Based on Multi-cores Environment[J]. Journal of Jilin University(Science Edition), 2014,52(1):59-64. 
[7] Zhou Weiming. Multicore computing and programming[M]. Wuhan: Huazhong University of Science and Technology Press, 2009.

[8] Luo Qiuming, Ming Zhong etc. OpenMP compiler theory and implementation technology [M].Beijing: Qinghua University Press,2012.

[9] Yan Weimin, Wu Weimin. Data Structure[M]. Beijing: Qinghua University Press, 1997. 